\title{
Non-Reciprocal Supratransmission in Mechanical Lattices with Non-Local Feedback Control Interactions
}

\author{
Jack E. Pechac and Michael J. Frazier *(D)
}

check for updates

Citation: Pechac, J.E.; Frazier, M.J. Non-Reciprocal Supratransmission in Mechanical Lattices with Non-Local Feedback Control Interactions. Crystals 2021, 11, 94. https:// doi.org/10.3390/cryst11020094

Received: 3 November 2020 Accepted: 20 January 2021 Published: 22 January 2021

Publisher's Note: MDPI stays neutral with regard to jurisdictional clai$\mathrm{ms}$ in published maps and institutional affiliations.

Copyright: (C) 2021 by the authors. Licensee MDPI, Basel, Switzerland. This article is an open access article distributed under the terms and conditions of the Creative Commons Attribution (CC BY) license (https:// creativecommons.org/licenses/by/ $4.0 /)$.
Department of Mechanical and Aerospace Engineering, University of California, San Diego, CA 92093, USA; jpechac@eng.ucsd.edu

* Correspondence: mjfrazier@ucsd.edu

\begin{abstract}
We numerically investigate the supratransmission phenomenon in an active nonlinear system modeled by the 1D/2D discrete sine-Gordon equation with non-local feedback. While, at a given frequency, the typical passive system exhibits a single amplitude threshold marking the onset of the phenomenon, we show that the inclusion of non-local feedback manifests additional thresholds that depend upon the specific boundary from which supratransmission is stimulated, realizing asymmetric (i.e., non-reciprocal) dynamics. The results illustrate a new means of controlling nonlinear wave propagation and energy transport for, e.g., signal amplification and mechanical logic.
\end{abstract}

Keywords: supratransmission; non-reciprocity; active mechanical lattices; sine-Gordon equation

\section{Introduction}

In the context of elastodynamics, phononic crystals and metamaterials [1,2]—collectively phononic materials - are two classes of materials whose artificial microstructure provides for the management of mechanical waves. For linear, small-amplitude waves, the microstructure design regulates internal scattering and resonance phenomena such that the Fourier components of a disturbance penetrate the material within only particular frequency ranges and in all or specific directions; outside these pass bands, i.e., within the band gaps, wave propagation is prohibited, the associated wave energy decaying exponentially in space. This filtering capability has inspired proposals for a variety of phononic material applications [3-5]. Nevertheless, nonlinearities inherent to the microstructure enable a unique dynamic response for large amplitude waves. Supratransmission describes the spontaneous flow of energy within the band gap via the nonlinear (non-topological) modes of a medium activated by a boundary driving of sufficient amplitude [6]. The effect is a generic property of nonlinear systems, having been shown to emerge from both integrable and non-integrable governing equations [7-12] even when accounting for dissipative effects [13] which more readily extinguish their linear counterparts. This may be exploited, e.g., for the transmission of binary, nonlinear signals in lightly-damped systems [14], as well as for the digital amplification of exceptionally weak signals for sensing $[9,12,15,16]$. While these and other studies have promoted an understanding of amplitude-dependent energy transmission, investigations of systems with a directional response, beneficial in applications for greater control of energy flow, are few.

Reciprocity describes the symmetry of wave transmission between two points in space: if a source and receiver exchange positions, the corresponding frequency response function is identical even in the presence of inhomogeneities and losses [17]. In recent years, inspired by the concept of electric [18] and optical $[19,20]$ diodes and motivated by their application in communications, sensing, and the directional control of energy flow, significant effort has been directed toward the discovery and study of non-reciprocity in other domains of physics. Regarding phononic materials [3,21-24], inherent nonreciprocity has been demonstrated in a number of systems utilizing unique and intersecting strategies for microstructure design, i.e., internal architectures characterized by internal 
motion [25,26], time-dependent [27-29] and topological [30-32] properties, and nonlinearity [33-35]. The focus of these and parallel studies is linear wave manipulation. Transition waves-nonlinear (topological) modes characteristic of multi-stable systems which propagate by liberating stored elastic energy-demonstrate non-reciprocity as well [36,37]; however, they are energetically limited to one-time operation or short propagation distances [38]. In the context of supratransmission, Wu et al. [39] exploited a spatial asymmetry in the metamaterial construction to elicit non-reciprocal transmission.

While most of the previous references employ a passive material platform, a small but growing collection $[25,28,29,31,32,34]$ investigate linear wave propagation within in an active setting. Recently, feedback control integrated directly into the material architecture has opened the door to more complex interactions unavailable in traditional structures and have been shown to be capable of eliciting non-reciprocal behavior [32,40]. Uniquely, rather than injecting energy into (or extracting from) the system by an external means, feedback involves observing the state of the system and then, following predetermined relations, generating a response that alters the present state. As a result, the material behavior is inherent rather than a function of environmental conditions (e.g., temperature, external fields, pumps, actuators which behave independently of the system). At present, the supratransmission phenomenon in feedback mediated nonlinear networks has yet to be investigated.

In this article, we present an approach to asymmetric (i.e., non-reciprocal) wave propagation in nonlinear mechanical networks with a focus on energy transmission within the band gap. Unique in the supratransmission literature, these lattice materials incorporate active elements which impart a local, non-conservative forcing proportional to non-local degrees-of-freedom. The result of this construction is that the onset of the supratransmission phenomenon is not only a function of the driving parameters (i.e., frequency and amplitude) but the specific network boundary at which the excitation is applied and from which wave energy is transmitted to the medium, establishing the asymmetric dynamic behavior.

The article is organized as follows: Section 2 presents the nonlinear governing equation of a representative, one-dimensional mechanical system with feedback and formulates the corresponding dispersion relation of linear, small-amplitude dynamics. In Section 3, we analyze the supratransmission characteristics of the system, demonstrating asymmetric performance. We also present results for a two-dimensional network. Section 4 concludes the article with a summary of the main results and proposals for future research directions.

\section{Theory}

\subsection{Model}

To demonstrate the feedback-mediated asymmetric energy transmission, we initially consider the nonlinear dynamics of a one-dimensional (1D) periodic network of coupled pendula, a modified version of that analyzed by Geniet and Leon [6] (Figure 1a). The pendulum motif is composed of a ring of radius, $\ell$, and mass, $m$, concentrated at a single point along the circumference. The pendulum rotates (in-plane), $\varphi$, about its center which, in the presence a gravitational field of strength $g$, adjusts the local potential as $\psi=m g \ell(1-\cos \varphi)$, which describes a non-convex energy landscape responsible for the network nonlinearity (Figure 1b). Moreover, the potential renders the system multi-stable, possessing several energetically degenerate ground states at $\varphi=2 \pi p, p \in \mathbb{Z}$. Utilizing elastic bands to manifest an effective (torsional) stiffness, $c=k \ell^{2}$, the network emerges via nearest-neighbor coupling. Uniquely, a feedback mechanism imposes an additional, non-conservative influence on each $\varphi_{j}$ which, from myriad possible descriptions, we relate to non-local variables; specifically, the torque, $f_{\mathrm{c}}$, applied to $\varphi_{j}$ via feedback is proportional to the relative rotation of its nearest neighbors, i.e., $f_{\mathrm{c}}=s\left(\varphi_{j+1}-\varphi_{j-1}\right)$, where $s$ denotes the proportional control gain. Physically, the feedback mechanism may emerge from active components which sense the non-local displacement and then, through a connected microcontroller and actuator, apply the calculated torque. For example, each pendulum may 
connect to a potentiometer which converts the angular displacement to a predetermined voltage drop measured by a programmable micro-controller. Dependent upon the voltage associated with $\varphi_{j+1}$ and $\varphi_{j-1}$, the micro-controller regulates a voltage sent to drive a DC motor which applies a torque, $f_{\mathrm{c}}$, to the pendulum at site $j$. For an arbitrary pendulum in the 1D network, the non-dimensional governing equation has the form

$$
\begin{aligned}
& \ddot{\varphi}_{j}+\bar{c}\left(2 \varphi_{j}-\varphi_{j+1}-\varphi_{j-1}\right) \\
& \quad+\bar{s}\left(\varphi_{j+1}-\varphi_{j-1}\right)+\bar{r} \sin \varphi_{j}=0
\end{aligned}
$$

where $\bar{c}=c \tau^{2} / m, \bar{s}=s \tau^{2} / m \ell^{2}$, and $\bar{r}=g \tau^{2} / m \ell$ are dimensionless parameters. The period of oscillation for an isolated pendulum, $\tau=2 \pi \sqrt{\ell / g}$, is a natural choice for the characteristic time and the normalizing parameter defining the dimensionless temporal variable, $\bar{t}=t / \tau$. Absent the feedback mechanism (i.e., $\bar{s}=0$ ), Equation (1) has a form that arises in several domains of science [41-43]; however, the sine-Gordon equation with feedback appears unique to the metamaterial described in this article.
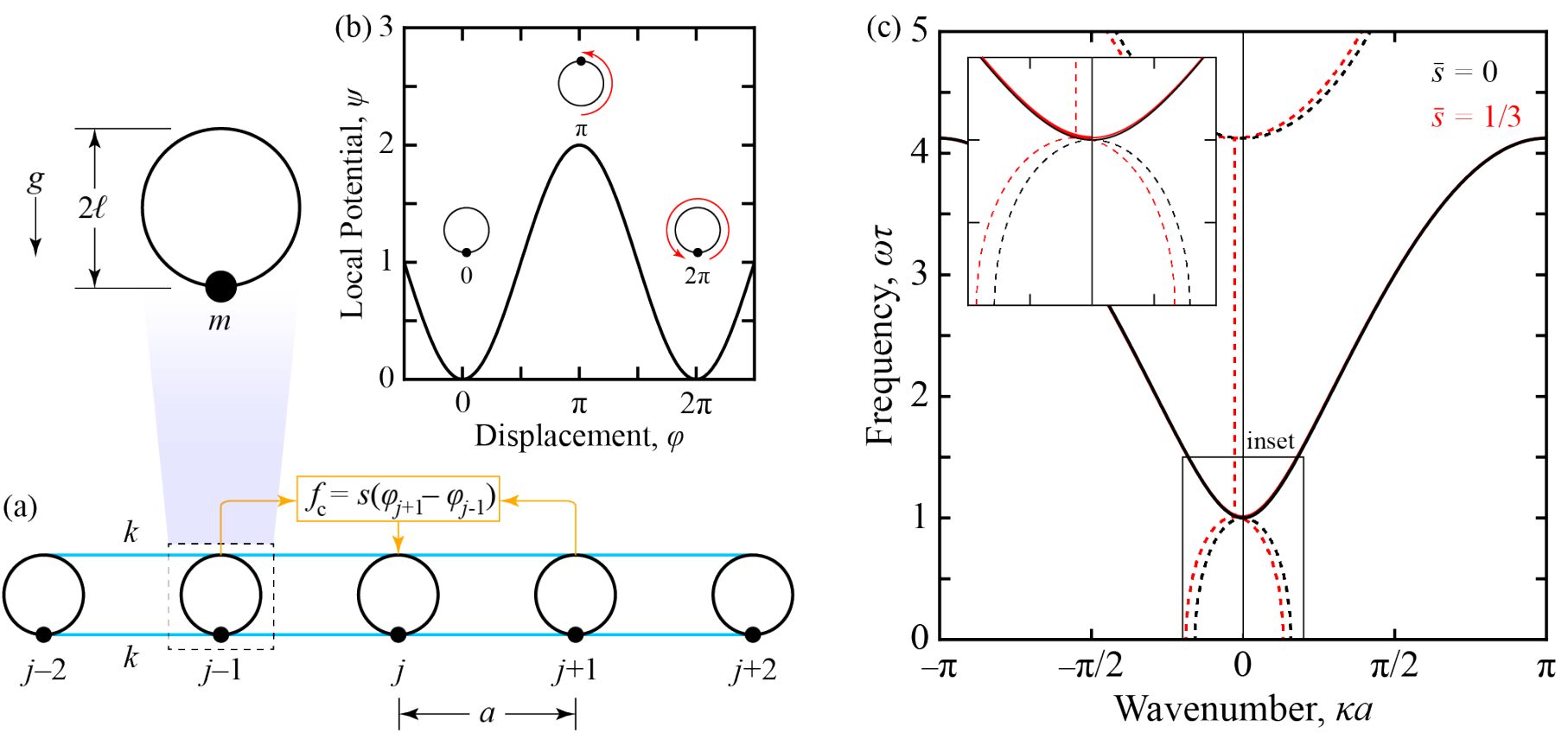

Figure 1. Pendulum network with feedback. (a) one-dimensional network of rotating pendula with elastic bands providing nearest-neighbor coupling and a feedback mechanism imposing a local influence which is proportional to the non-local displacements, $f_{\mathrm{c}}=s\left(\varphi_{j+1}-\varphi_{j-1}\right)$; (b) the on-site, multi-stable potential which manifests the system nonlinearity; (c) the complex dispersion of small-amplitude waves of prescribed non-dimensional frequency, $\omega \tau$, with real and imaginary wavenumbers, $\kappa_{\mathrm{R}}$ (solid) and $\kappa_{\mathrm{I}}$ (dotted), respectively. The feedback effect shifts the attenuating structure about $\kappa_{\mathrm{I}}=0$, provoking non-reciprocal wave propagation.

For a system of $N$ pendula, Equation (1) forms the basis of a system of equations, $\mathbf{M} \ddot{\mathbf{u}}+(\mathbf{K}+\mathbf{S}) \mathbf{u}+\mathbf{f}_{\mathrm{NL}}=\mathbf{0}$, where $\mathbf{M}, \mathbf{K}$, and $\mathbf{S}$, respectively, are the mass, stiffness, and feedback matrices; $\mathbf{u}$ and $\mathbf{f}_{\mathrm{NL}}$ are, accordingly, the displacement and nonlinear force vectors. While the mass and stiffness matrices are symmetric, $\mathbf{S} \neq \mathbf{S}^{\mathrm{T}}$, which renders the system non-Hermitian and supportive of non-reciprocal dynamics. Alternatively, $\mathbf{K}+\mathbf{S}$ may emerge from a system in which the spring interactions, when deformed, manifest unequal forces at each end. Moreover, from the continuum limit of Equation (1) with expansion $\varphi_{j \pm 1} \rightarrow \varphi \pm \varphi_{, \bar{x}}+\varphi_{, \bar{x} \bar{x}} / 2$,

$$
\varphi_{, \bar{t} \bar{t}}-\bar{c} \varphi_{, \bar{x}} \bar{x}+2 \bar{s} \varphi_{, \bar{x}}+\bar{r} \sin \varphi_{j}=0,
$$


where $\bar{x}=x / a$, it is apparent that the activity either injects or extracts energy (spatially) from the system depending on the sign of $\bar{s} \varphi, x$. Consequently, for a positive $\bar{s}$ and, e.g., an exponentially decreasing (increasing) displacement function, the active term tends to support (dampen) the motion of $\varphi$. Odd spatial derivatives of higher order would have a similar effect.

\subsection{Dispersion}

The proposed system supports a number of amplitude-dependent solutions owing to the nonlinear on-site potential. For the particular case of small-amplitude motion, the Fourier components of an initially compact disturbance disperse as the propagation speeds depend on the corresponding wavelength. To establish the dispersion relation, we first linearize Equation (1) about a (stable) equilibrium configuration and then theoretically extend the pendulum network to infinity through the application of Bloch boundary conditions on a single unit cell; thus, the dynamics of such a system are described by $\varphi_{j}=\Phi \mathrm{e}^{\mathrm{i}(\kappa j a-\omega \tau \bar{t})}$ with $\kappa$ and $\omega$, respectively, the spatial and temporal frequencies. Applied to Equation (1), the Bloch wave solution entails the following dispersion relation:

$$
-\omega^{2} \tau^{2}+\bar{c}(2-\gamma-1 / \gamma)+\bar{s}(\gamma-1 / \gamma)+\bar{r}=0,
$$

where $\gamma=\mathrm{e}^{\mathrm{i} \kappa a}$. The complex band structure emerges from the solution, $\gamma(\omega \tau)$, which contains the real and imaginary components of the normalized wavenumber, $\kappa a=\kappa_{\mathrm{R}} a+\mathrm{i} \kappa_{\mathrm{I}} a$ : $\kappa_{\mathrm{R}} a=\operatorname{Re}[-\mathrm{i} \ln (\gamma)]$, specifying the spatial oscillation of displacement, and $\kappa_{\mathrm{I}} a=\operatorname{Im}[-\mathrm{i} \ln (\gamma)]$, expressing the spatial attenuation of the amplitude.

\section{Results}

\subsection{Simulation}

In the following, we numerically investigate the dynamics of a representative nonlinear network with non-local feedback. To this end, we consider a finite, nonlinear system of $N$ pendula subject to a prescribed harmonic boundary displacement, $\varphi_{j}=\Phi \sin (\omega \tau \bar{t})$, where $\omega \tau$ is set within the band gap and $\Phi$ varies between simulations. The left (L) and right (R) boundaries are distinguished, respectively, by $j=1$ and $j=N$. The system response is quantified by the mean energy transmitted to the network by the driven boundary over an $n \in \mathbb{Z}^{+}$multiple of the excitation period, $T$ :

$$
E_{\mathrm{in}}=\frac{\bar{c}}{n T} \int_{0}^{n T}\left(\varphi_{2}-\varphi_{1}\right) \dot{\varphi}_{1} \mathrm{~d} \bar{t}
$$

for the left boundary; for the right boundary, substitute the indices in Equation (3) as $\{1,2\} \rightarrow\{N, N-1\}$. For sufficiently small amplitudes, the system exhibits a linear response where the energy supplied to the system, ultimately, returns to the driving due to Bragg reflection, causing $E_{\mathrm{in}}$ to vanish. Conversely, beyond a critical amplitude, $\Phi_{\mathrm{c}}$, the driving excites nonlinear modes which penetrate the system, resulting in $E_{\text {in }}>0$, signifying a spontaneous energy flow. While a number of methods have been developed to predict the amplitude threshold [7,44-47], in this article, we intend to introduce the concept of active lattices to the supratransmission literature and initiate exploration of its effects.

Simulations evolve Equation (1) for a network of $N=1000$ coupled pendula with $\bar{c}=4$ and $\bar{r}=1$ for $n=100$ periods. In order to minimize reflections from the free boundary (thus, mimicking an infinite medium), we apply a linearly increasing viscous damping, $\bar{\eta} \dot{\varphi}_{j}$, to the final 800 sites with $\max (\bar{\eta})=1 / 10$. In order to avoid the shock wave generated by vanishing initial velocities, the simulations adopt an inaugural velocity profile matching the (linear) evanescent solution at the driving frequency, i.e., $\dot{\varphi}_{j}=-\omega \tau \Phi \cos [(j-$ 1) $\left.\kappa_{\mathrm{R}} a\right] \mathrm{e}^{-(j-1) \kappa_{\mathrm{I}} a}$. In simulation, each boundary is excited in turn, revealing a directionspecific energy transmission for $\bar{s} \neq 0$. 


\subsection{Supratransmission: One Dimension}

Figure 1c graphs the dispersion diagram for instances of passive $(\bar{s}=0)$ and active $(\bar{s}=1 / 3)$ feedback control, each exhibiting a single pass band separating two band gaps. Apparently, for the reference case where $\bar{s}=0$, the diagram is symmetric about $\mathcal{K}=0$, implying that wave propagation is independent of direction. Harmonic boundary driving in the pass band transmits wave energy unabated into the network; in the band gap, Bragg reflection confines energy to the boundary as indicated by $\kappa_{\mathrm{I}} \neq 0$. Activating the feedback mechanism modifies the dispersion. While the real component of the band structure, $\kappa_{R}(\omega \tau)$, remains symmetric about $\kappa=0$, the imaginary component, $\kappa_{\mathrm{I}}(\omega \tau)$, shifts primarily along the wavenumber axis, breaking the diagram symmetry. This implies that wave propagation, including the supratransmission phenomenon, is direction-dependent under non-local feedback control. This is because the onset of supratransmission is linked to the critical amplitude which destabilizes the evanescent wave profile [48], whose feedback has not been made direction-dependent. Analyzing small-amplitude waves, Rosa and Ruzzene [32] also observed non-reciprocal behavior via a feedback effect that was attributed to a complex temporal frequency whose imaginary component resulted in a propagation direction dependent exponential growth or decay. This article describes a similar effect for nonlinear waves which is attributed to a shift in the imaginary wavenumber component of the linear wave dispersion.

Figure 2a plots the energy transmission efficiency of a harmonic boundary driving within the lower band gap $(\omega \tau=0.9)$ in the pendulum network as a function of the driving amplitude for two values of the control gain, $\bar{s}=0$ and $\bar{s}=1 / 3$. In general, below a critical amplitude, $\Phi_{c}$, the linear, small-amplitude response of the system dominates as the total energy remains concentrated near the driven boundary. As the driving amplitude increases, however, higher harmonic modes-the multiples of the driving frequency which appear in the pass band-generated by burgeoning nonlinear effects propagate into the system, contributing to a relatively small increase in $E_{\text {in }}$. For the reference case $(\bar{s}=0)$, beyond the critical amplitude predicted by Geniet and Leon [6], $\Phi_{\mathrm{c}}=1.77$, the attenuating displacement profile of the linear solution is unstable [48,49]; instead, nonlinear modes generated at the driven boundary subsequently propagate into the system, signified by the sudden increase in the transmitted energy, i.e., supratransmission. As the band diagram of the reference system is symmetric (Figure 1c), the critical amplitude for supratransmission is identical for left- and right-boundary driving. However, setting $\bar{s} \neq 0$ activates the feedback mechanism and adjusts the band diagram as described previously-for $\bar{s}=1 / 3$ and $\omega \tau=0.9$, forward and backward waves are attenuated according to $\kappa_{\mathrm{I}} a=0.150$ and $\kappa_{\mathrm{I}} a=0.317$, respectively-which suggests that $\Phi_{\mathrm{c}}$ differs for the same excitation on opposite boundaries. The simulation results depicted in Figure 2a confirm that the amplitude thresholds for supratransmission in the feedback network do, indeed, differ: $\Phi_{\mathrm{c}}^{\mathrm{L}}=1.41$ and $\Phi_{\mathrm{c}}^{\mathrm{R}}=2.44$, respectively, for left- and right-boundary driving. Figure $2 \mathrm{~b}$ shows how the two thresholds diverge as a function of $\bar{s}$.

The supratransmission demonstrated in the previous results transmits the energy of a signal with band-gap frequency through a nonlinear system. To further emphasize this phenomenon, Figure $2 \mathrm{c}$ displays snapshots of the left and right boundary regions of the active pendula network $(\bar{s}=1 / 100)$ subject to the same harmonic driving with various amplitudes. For visualization purposes, the instantaneous displacement in the boundary region is normalized such that $\max \left(\left|\varphi_{j}\right|\right)=1$.

The superposed color indicates the corresponding energy distribution over the same region, $\mathcal{H}_{j}=\frac{1}{2} \dot{\varphi}_{j}^{2}+\frac{1}{2} \bar{c}\left(\varphi_{j}-\varphi_{j-1}\right)^{2}+\psi\left(\varphi_{j}\right)$. As expected, for small amplitudes, the displacement profile exhibits an exponential decay and energy concentrates at the boundary. In the second row of Figure 2c, the excitation amplitude exceeds the supratransmission threshold for only the left boundary driving; consequently, the evanescent response is unstable and energy propagates away from the boundary. The same excitation amplitude does not exceed the critical value for the right boundary; therefore, apart from the aforementioned higher harmonics lying within the pass band, the energy remains localized there. 
(a)
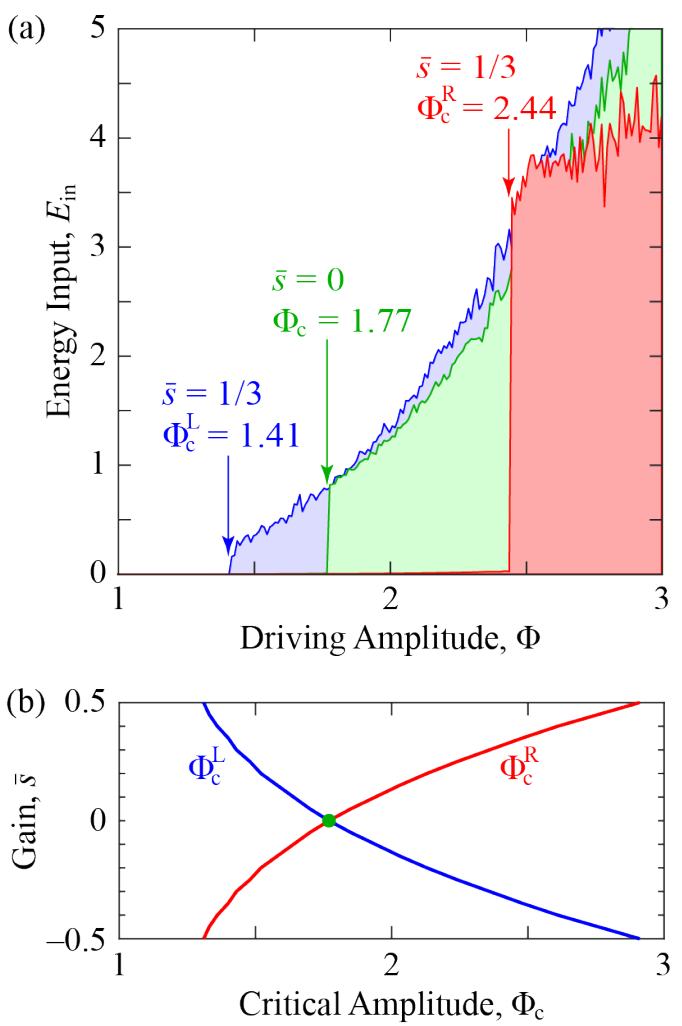

(c)
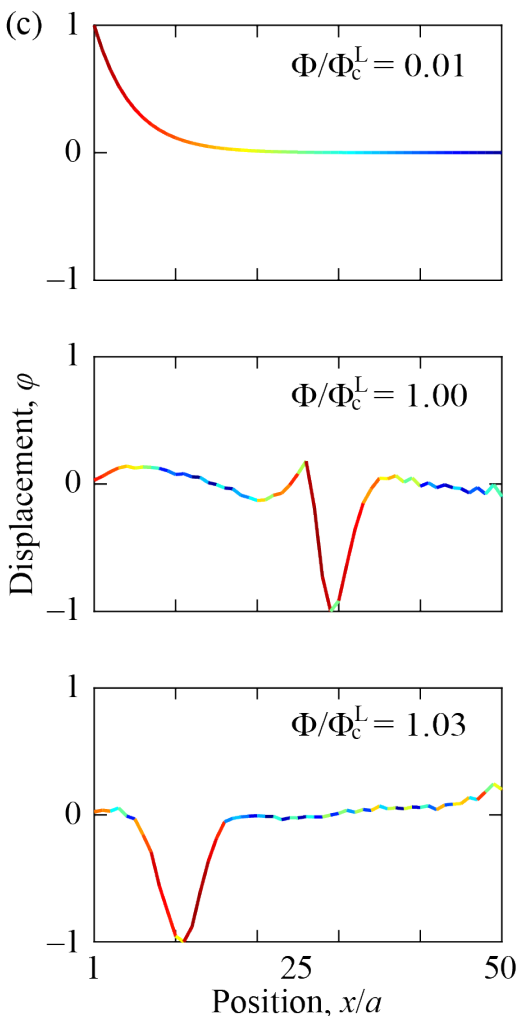
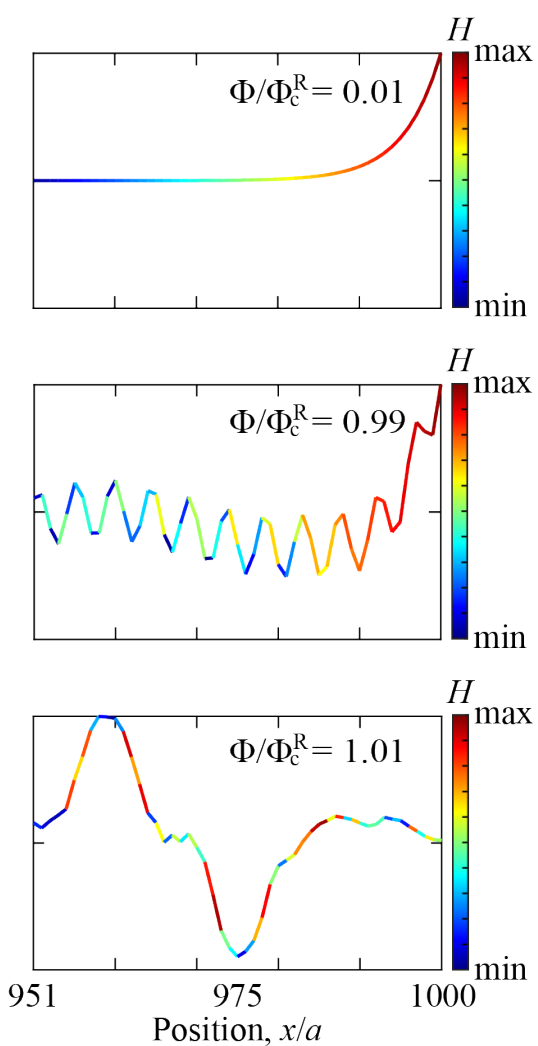

Figure 2. Asymmetric supratransmission in one dimension (color online). (a) energy transmission as a function of the driving amplitude. For supratransmission, the passive system $(\bar{s}=0)$ exhibits a common threshold amplitude, $\Phi_{\mathrm{c}}=1.77$, when excited from either the left or right boundary; conversely, the active system $(\bar{s}=1 / 3)$ expresses two thresholds, $\Phi_{\mathrm{c}}^{\mathrm{L}}=1.41$ and $\Phi_{\mathrm{c}}^{\mathrm{R}}=2.44$, when excited from the left and right, respectively; (b) the separation of $\Phi_{\mathrm{c}}^{\mathrm{L}}$ and $\Phi_{\mathrm{c}}^{\mathrm{R}}$ over a range of $\bar{s}$; (c) the normalized displacement profile and energy distribution for an active system $(\bar{s}=1 / 100)$ at various amplitudes. For each row, the left and right boundaries are subject to the same harmonic excitation, the amplitude of which may be above/below the critical amplitude to trigger supratransmission at the particular boundary.

\subsection{Supratransmission: Two Dimensions}

The pendulum network can be extended to obtain a two-dimensional (2D) periodic system, e.g., a square lattice with nearest-neighbor coupling. Accordingly, the non-local feedback applied to each $\varphi_{j, k}$ depends on additional terms, $f_{\mathrm{c}}=\bar{s}_{1}\left(\varphi_{j+1, k}-\varphi_{j-1, k}\right)+$ $\bar{s}_{2}\left(\varphi_{j, k+1}-\varphi_{j, k-1}\right)$ where $\bar{s}_{1}$ and $\bar{s}_{2}$ are gain parameters. Considering a Cartesian frame, indices $j$ and $k$ designate sites along the $x$-and $y$-axes, respectively, with the indices increasing with the relevant coordinate. Following the same normalization scheme as before, the governing equation for a generic unit cell is

$$
\begin{aligned}
\ddot{\varphi}_{j, k} & +\bar{c}\left(4 \varphi_{j, k}-\varphi_{j+1, k}-\varphi_{j-1, k}-\varphi_{j, k+1}-\varphi_{j, k-1}\right) \\
& +\bar{s}_{1}\left(\varphi_{j+1, k}-\varphi_{j-1, k}\right)+\bar{s}_{2}\left(\varphi_{j, k+1}-\varphi_{j, k-1}\right) \\
& +\bar{r} \sin \varphi_{j, k}=0 .
\end{aligned}
$$

Linearizing Equation (4) and applying the Bloch solution, $\varphi_{j, k}=\Phi \mathrm{e}^{\mathrm{i}\left(\kappa_{\mathrm{x}} j a+\kappa_{\mathrm{y}} k a-\omega \tau \bar{t}\right)}$, yields the dispersion relation

$$
\begin{aligned}
-\omega^{2} \tau^{2} & +\bar{c}\left(4-\gamma_{\mathrm{x}}-1 / \gamma_{\mathrm{x}}-\gamma_{\mathrm{y}}-1 / \gamma_{\mathrm{y}}\right) \\
& +\bar{s}_{1}\left(\gamma_{\mathrm{x}}-1 / \gamma_{\mathrm{x}}\right)+\bar{s}_{2}\left(\gamma_{\mathrm{y}}-1 / \gamma_{\mathrm{y}}\right)+\bar{r}=0
\end{aligned}
$$

where $\gamma_{\mathrm{x}}=\mathrm{e}^{\mathrm{i} \kappa_{\mathrm{x}} a}$ and $\gamma_{\mathrm{y}}=\mathrm{e}^{\mathrm{i} \kappa_{\mathrm{y}} a}$. Figure $3 \mathrm{a}$ plots the complex dispersion surfaces for the passive system $\left(\bar{s}_{1}=\bar{s}_{2}=0\right)$, revealing a single propagating surface separating two 
attenuating surfaces within frequency band gaps. Since, as before, our focus is in the lower band gap where the real component of the wavevector vanishes, we substitute $\gamma_{\mathrm{x}}=\mathrm{e}^{-\kappa_{\mathrm{I}, \mathrm{x}} a}$ and $\gamma_{\mathrm{y}}=\mathrm{e}^{-\kappa_{\mathrm{I}, \mathrm{y}} a}$ in Equation (5) such that, for a given $\omega \tau$ in the lower band gap, the $\kappa_{\mathrm{I}, \mathrm{x}}$ and $\kappa_{\mathrm{I}, \mathrm{y}}$ which satisfy the relation trace the circular contour of the attenuating surface. Specifically, at the band-gap frequency $\omega \tau=0.9$, the diagrams in Figure $3 \mathrm{~b}$ compare the attenuating contours of various active systems with that of the passive lattice. Apparently, similar to the earlier 1D system, in the 2D network, activity causes the attenuating contour to shift relative to the passive reference centered at $\left(\kappa_{\mathrm{x}}, \kappa_{\mathrm{y}}\right)=(0,0)$, indicating the broken symmetry of the system's small-amplitude dynamics which we expect to persist at larger amplitudes.

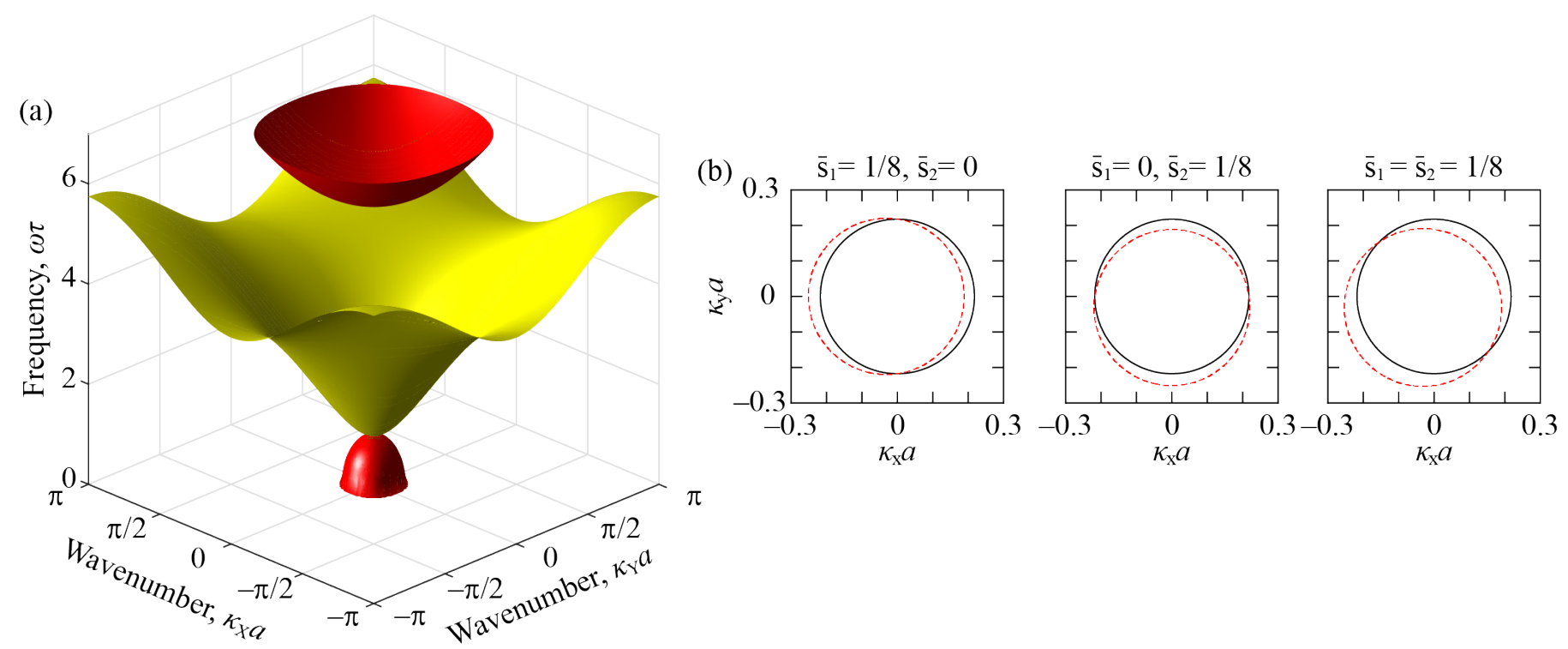

Figure 3. Dispersion (color online). (a) the complex dispersion surfaces for a passive, square lattice of pendula with near-neighbor interactions, including the propagating (yellow) and attenuating (red) modes; (b) the circular contour formed by the cross-section of the attenuating surface at band-gap frequency $\omega \tau=0.9$. Active feedback, i.e., $\bar{s}_{1,2} \neq 0$, shifts the attenuation contour (red, dashed) with respect to the passive result (black), enabling direction-dependent non-reciprocal wave propagation.

To investigate asymmetric supratransmission in the 2D setting, we track the energy transmitted to a square lattice of finite dimension by a set of co-linear sites oscillating in phase at a band-gap frequency (Figure 4a). Beyond a critical amplitude, the driven boundary generates a wave front which propagates away from the driving and normal to it, i.e., at an angle $\theta$ with respect to the $x$-axis. Thus, the directional dependence of the supratransmission phenomena may be investigated by adjusting the slope of the driven boundary. To this end, we modify Equation (3) to accommodate the additional interactions of the 2D system under consideration:

$$
E_{\text {in }}=\frac{\bar{c}}{n T} \sum_{m, n} \sum_{r, s} \int_{0}^{n T}\left(\varphi_{r, s}-\varphi_{m, n}\right) \dot{\varphi}_{m, n} \mathrm{~d} \bar{t},
$$

where $\{m, n\}$ collects the indices of driven sites and $\{r, s\}$ the indices of their nearest neighbor(s). Thus, Equation (6) is the time-averaged energy transmitted to the lattice by the driven boundary.

Simulations utilize a square lattice with nearest-neighbor interactions to develop a rectangular system of approximately $23 a \times 800 a$ in dimension with the driven sites along one of the smaller dimensions and periodic boundary conditions along the two extended dimensions. For $\bar{c}=4$ and $\bar{r}=1$, the excitation frequency $\omega \tau=0.9$ lies within the band gap. For $n=25$ periods, we evolve the system following Equation (4) from an initial 
velocity, $\dot{\varphi}_{j, k}=-\omega \tau \Phi \cos \left(\kappa_{\mathrm{R}} d\right) \mathrm{e}^{\kappa_{\mathrm{I}} d}$, where $d$ is the perpendicular distance from the driven boundary to the $(j, k)$ th site and $\kappa=\kappa_{\mathrm{R}}+\mathrm{i} \kappa_{\mathrm{I}}$ is the complex wavenumber in that direction.

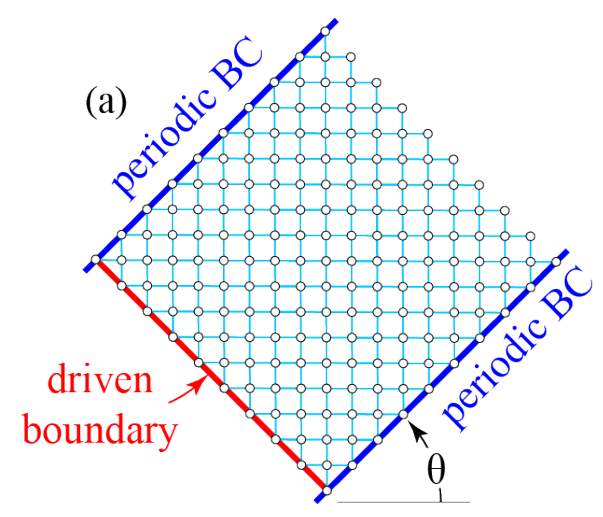

(b)

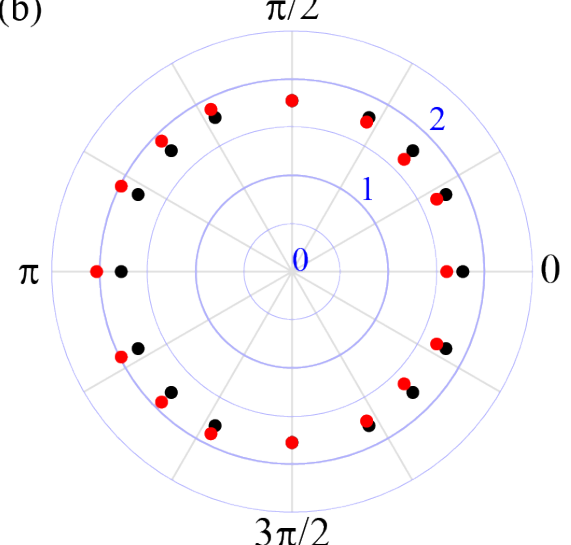

$\bar{S}_{1}=1 / 8, \bar{S}_{2}=0$
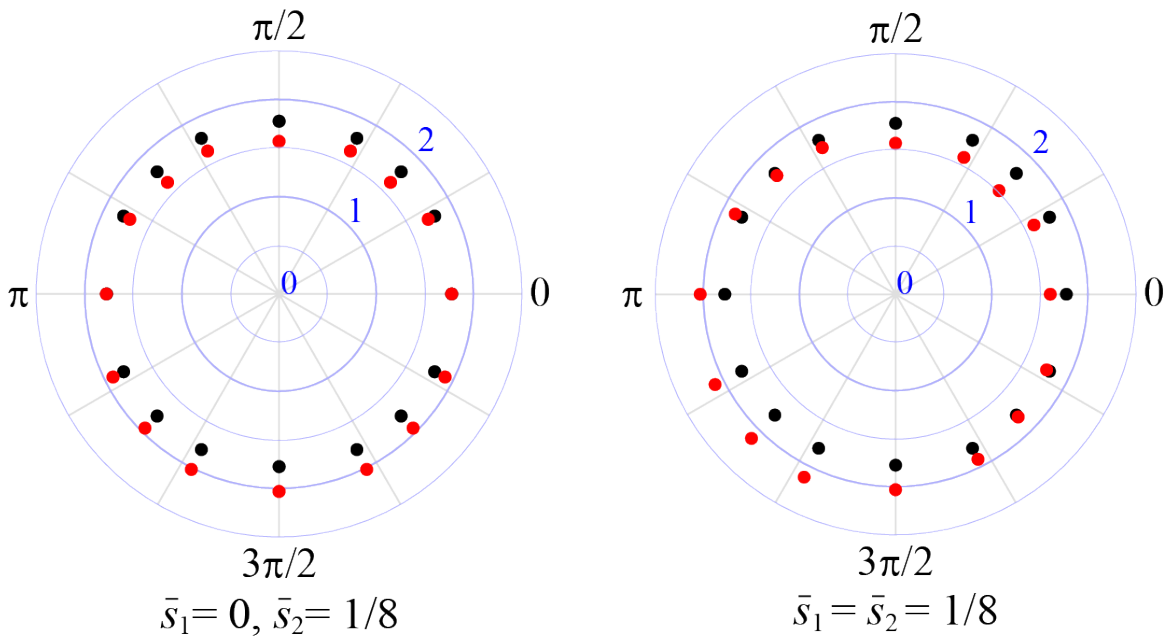

Figure 4. Asymmetric supratransmission in two dimensions (color online). (a) finite square lattice with periodic boundary conditions (blue) and a harmonically exciting line of nodes (red). (b) The threshold amplitude $\Phi_{c}$ versus orientation angle $\theta$ reveals the directional impact of activity for different combinations of the feedback parameters (red) relative to a passive system (black).

For a number of orientations, $\theta$, we determine the critical driving amplitude above which $E_{\text {in }}$ sharply increases. The panels in Figure $4 \mathrm{~b}$ display the directional dependence of the threshold amplitude for different combinations of the feedback parameters, $\bar{s}_{1}$ and $\bar{s}_{2}$. For the passive system, we determine a $\theta$-independent threshold amplitude of $\Phi_{\mathrm{c}}=1.77$ that is identical to that of the 1D passive system. However, following the wavenumber contours in Figure 3b, activity causes the threshold amplitude to shift relative to its passive value, the magnitude of the shift dependent upon $\theta$. For the active scenario $\left(\bar{s}_{1}, \bar{s}_{2}\right)=(1 / 8,0)$, the minimum amplitude for supratransmission decreases for waves propagating to the right (i.e., $|\theta|<\pi / 2$ ) and increases for waves traveling to the left (i.e., $|\theta|>\pi / 2$ ). The effect is maximized for $\theta=0$ where the wave front aligns with the gradient, $\varphi_{r}$, which determines the strength of $f_{\mathrm{c}}$. Conversely, the threshold is unchanged at $\theta= \pm \pi / 2$ where the effect of $\varphi_{, x}$ and, therefore, $f_{\mathrm{c}}$ vanish. Figure $4 \mathrm{~b}$ displays additional orientation-amplitude results for systems with alternative feedback definitions that may be similarly understood.

\section{Conclusions}

To summarize, we numerically investigated the nonlinear supratransmission phenomenon in active $1 \mathrm{D} / 2 \mathrm{D}$ periodic networks characterized by a non-local feedback control. We found that such a feedback adjusts the imaginary wavenumber component across the 
whole of the frequency range, although asymmetrically, such that the otherwise reciprocal dynamics becomes direction-dependent. In the context of band gap energy transmission in finite, one-dimensional networks, the critical amplitude for stimulating supratransmission via boundary excitation differs for each boundary. Similarly, for a two-dimensional system, the amplitude threshold changes with boundary orientation. These results demonstrate an alternative, extremely tunable approach toward non-reciprocal dynamics and applications $[9,14]$.

There are several research directions for subsequent studies. The influence of anisotropy and lattice type, especially those with multiple elements per lattice site (e.g., Lieb, Kagome, etc.), on the directionality of supratransmission is worth investigating. While we have considered a proportional controller with displacement inputs, a natural extension of the present work would explore controllers of derivative- and integral-type to determine the characteristics of each feedback archetype, independently followed by in combination. In addition, feedback definitions involving spatial and temporal rates have yet to be explored.

Author Contributions: Conceptualization, M.J.F.; formal analysis, J.E.P. and M.J.F.; investigation, J.E.P. and M.J.F.; methodology, M.J.F.; project administration, M.J.F.; supervision, M.J.F.; validation, J.E.P. and M.J.F.; visualization, J.E.P. and M.J.F.; writing—original draft, M.J.F.; writing-review \& editing, J.E.P. and M.J.F. All authors have read and agreed to the published version of the manuscript.

Funding: This research was funded by the University of California.

Conflicts of Interest: The authors declare no conflict of interest.

\section{References}

1. Hussein, M.I.; Leamy, M.J.; Ruzzene, M. Dynamics of phononic materials and structures: historical origins, recent progress, and future outlook. Appl. Mech. Rev. 2014, 66, 040802. [CrossRef]

2. Ma, G.; Sheng, P. Acoustic metamaterials: From local resonances to broad horizons. Sci. Adv. 2016, 2. [CrossRef] [PubMed]

3. Li, X.F.; Ni, X.; Feng, L.; Lu, M.H.; He, C.; Chen, Y.F. Tunable unidirectional sound propagation through a sonic-crystal-based acoustic diode. Phys. Rev. Lett. 2011, 106, 084301. [CrossRef] [PubMed]

4. Zhong, J.; Zhao, H.; Yang, H.; Wang, Y.; Yin, J.; Wen, J. Theoretical requirements and inverse design for broadband perfect absorption of low-frequency waterborne sound by ultrathin metasurface. Sci. Rep. 2019, 9. [CrossRef]

5. Benichou, I.; Givli, S. Force-sensitive metamaterials for vibration mitigation and mechanical protection. Extreme Mech. Lett. 2020, 40. [CrossRef]

6. Geniet, F.; Leon, J. Energy transmission in the forbidden band gap of a nonlinear chain. Phys. Rev. Lett. 2002, 89, 134102. [CrossRef] [PubMed]

7. Khomeriki, R.; Lepri, S.; Ruffo, S. Nonlinear supratransmission and bistability in the Fermi-Pasta-Ulam model. Phys. Rev. E 2004, 70, 066626. [CrossRef] [PubMed]

8. Macías-Díaz, J.E. Numerical study of the transmission of energy in discrete arrays of sine-Gordon equations in two space dimensions. Phys. Rev. E 2008, 77, 016602. [CrossRef] [PubMed]

9. Macías-Díaz, J.E. Bistability of a two-dimensional Klein-Gordon system as a reliable means to transmit monochromatic waves: A numerical approach. Phys. Rev. E 2008, 78, 056603. [CrossRef]

10. Togueu Motcheyo, A.B.; Tchawoua, C.; Tchinang Tchameu, J.D. Supratransmission induced by waves collisions in a discrete electrical lattice. Phys. Rev. E 2013, 88, 040901. [CrossRef] [PubMed]

11. Yousefzadeh, B.; Phani, A.S. Energy transmission in finite dissipative nonlinear periodic structures from excitation within a stop band. J. Sound Vib. 2015, 354, 180-195. [CrossRef]

12. Malishava, M. All-phononic amplification in coupled cantilever arrays based on gap soliton dynamics. Phys. Rev. E 2017, 95 . [CrossRef] [PubMed]

13. Geniet, F.; Leon, J. Nonlinear supratransmission. J. Phys. Condens. Matter 2003, 15, 2933-2949. [CrossRef]

14. Macías-Díaz, J.E.; Puri, A. An application of nonlinear supratransmission to the propagation of binary signals in weakly damped, mechanical systems of coupled oscillators. Phys. Lett. A 2007, 366, 447-450. [CrossRef]

15. Khomeriki, R.; Leon, J.; Chevriaux, D. Quantum Hall bilayer digital amplifier. Eur. Phys. J. B 2006, 49, 213-218. [CrossRef]

16. Malishava, M.; Khomeriki, R. All-phononic digital transistor on the basis of gap-soliton dynamics in an anharmonic oscillator ladder. Phys. Rev. Lett. 2015, 115, 104301. [CrossRef]

17. Nassar, H.; Yousefzadeh, B.; Fleury, R.; Ruzzene, M.; Alu, A.; Daraio, C.; Norris, A.N.; Huang, G.; Haberman, M.R. Nonreciprocity in acoustic and elastic materials. Nat. Rev. Mater. 2020, 5. [CrossRef]

18. Matsuo, H.; Kitanaka, Y.; Inoue, R.; Noguchi, Y.; Miyayama, M. Switchable diode-effect mechanism in ferroelectric BiFeO 3 thin film capacitors. J. Appl. Phys. 2017, 118, 114101. [CrossRef]

19. Potton, R.J. Reciprocity in optics. Rep. Prog. Phys. 2004, 67, 717-754. [CrossRef] 
20. Kodera, T.; Sounas, D.L.; Caloz, C. Magnetless nonreciprocal metamaterial (MNM) technology: application to microwave components. IEEE Trans. Microw. Theory Tech. 2013, 61, 1020-1042. [CrossRef]

21. Liang, B.; Yuan, B.; Cheng, J.C. Acoustic diode: rectification of acoustic energy flux in one-dimensional systems. Phys. Rev. Lett. 2009, 103, 104301. [CrossRef] [PubMed]

22. Ma, C.; Parker, R.G.; Yellen, B.B. Optimization of an acoustic rectifier for uni-directional wave propagation in periodic mass-spring lattices. J. Sound Vib. 2013, 332, 4876-4894. [CrossRef]

23. Maznev, A.A.; Every, A.G.; Wright, O.B. Reciprocity in reflection and transmission: What is a 'phonon diode'? Wave Motion 2013, 50, 776-784. [CrossRef]

24. Fleury, R.; Sounas, D.L.; Haberman, M.R.; Alu, A. Nonreciprocal acoustics. Acoust. Today 2015, 11, $14-21$.

25. Fleury, R.; Sounas, D.L.; Sieck, C.F.; Haberman, M.R.; Alu, A. Sound isolation and giant linear nonreciprocity in a compact acoustic circulator. Science 2014, 343, 516-519. [CrossRef]

26. Attarzadeh, M.A.; Callanan, J.; Nouh, M. Experimental observation of nonreciprocal waves in a resonant metamaterial beam. Phys. Rev. Appl. 2020, 13, 021001. [CrossRef]

27. Swinteck, N.; Matsuo, S.; Runge, K.; Vasseur, J.O.; Lucas, P.; Deymier, P.A. Bulk elastic waves with unidirectional backscatteringimmune topological states in a time-dependent superlattice. J. Appl. Phys. 2015, 118, 063103. [CrossRef]

28. Wang, Y.; Yousefzadeh, B.; Chen, H.; Nassar, H.; Huang, G.; Daraio, C. Observation of nonreciprocal wave propagation in a dynamic phononic lattice. Phys. Rev. Lett. 2018, 121, 194301. [CrossRef]

29. Trainiti, G.; Xia, Y.; Marconi, J.; Cazzulani, G.; Erturk, A.; Ruzzene, M. Time-periodic stiffness modulation in elastic metamaterials for selective wave filtering: theory and experiment. Phys. Rev. Lett. 2019, 122, 124301. [CrossRef]

30. Pal, R.K.; Schaeffer, M.; Ruzzene, M. Helical edge states and topological phase transitions in phononic systems using bi-layered lattices. J. Appl. Phys. 2016, 119, 084305. [CrossRef]

31. Souslov, A.; van Zuiden, B.C.; Bartolo, D.; Vitelli, V. Topological sound in active-liquid metamaterials. Nat. Phys. 2017, 13, 1091-1094. [CrossRef]

32. Rosa, M.I.N.; Ruzzene, M. Dynamics and topology of non-Hermitian elastic lattices with non-local feedback control interactions. New J. Phys. 2020, 22, 053004. [CrossRef]

33. Liang, B.; Guo, X.S.; Tu, J.; Zhang, D.; Cheng, J.C. An acoustic rectifier. Nat. Mat. 2010, 9, 989-992. [CrossRef] [PubMed]

34. Popa, B.I.; Cummer, S.A. Non-reciprocal and highly nonlinear active acoustic metamaterials. Nat. Commun. 2014, 5, 4398. [CrossRef] [PubMed]

35. Raney, J.R.; Nadkarni, N.; Daraio, C.; Kochmann, D.M.; Lewis, J.A.; Bertoldi, K. Stable propagation of mechanical signals in soft media using stored elastic energy. Proc. Natl. Acad. Sci. USA 2016, 113, 9722-9727. [CrossRef]

36. Nadkarni, N.; Arrieta, A.F.; Chong, C.; Kochmann, D.M.; Daraio, C. Unidirectional transition waves in bistable lattices. Phys. Rev. Lett. 2016, 116, 244501. [CrossRef] [PubMed]

37. Hwang, M.; Arrieta, A.F. Solitary waves in bistable lattices with stiffness grading: augmenting propagation control. Phys. Rev. E 2018, 98, 042205. [CrossRef]

38. Ramakrishnan, V.; Frazier, M.J. Transition waves in multi-stable metamaterials with space-time modulated potentials. Appl. Phys. Lett. 2020, 117, 151901. [CrossRef]

39. Wu, Z.; Zheng, Y.; Wang, K.W. Metastable modular metastructures for on-demand reconfiguration of band structures and nonreciprocal wave propagation. Phys. Rev. E 2018, 97, 022209. [CrossRef]

40. Brandenbourger, M.; Locsin, X.; Lerner, E.; Coulais, C. Non-reciprocal robotic metamaterials. Nat. Commun. 2019, 10, 4608. [CrossRef]

41. Frenkel, Y.I.; Kontorova, T. On the theory of plastic deformation and twinning. J. Phys. 1939, 1, 137-149.

42. Perring, J.K.; Skyrme, T.H.R. A model unified field equation. Nucl. Phys. 1962, 31. [CrossRef]

43. Josephson, B.D. Supercurrents through barriers. Adv. Phys. 1965, 14, 419-451. [CrossRef]

44. Kopidakis, G.; Aubry, S. Discrete breathers and delocalization in nonlinear disordered systems. Phys. Rev. Lett. 2000, 84, 3236-3239. [CrossRef] [PubMed]

45. Maniadis, P.; Kopidakis, G.; Aubry, S. Energy dissipation threshold and self-induced transparency in systems with discrete breathers. Phys. D 2006, 216, 121-135. [CrossRef]

46. Wu, Z.; Wang, K.W. On the wave propagation analysis and supratransmission prediction of a metastable modular metastructure for non-reciprocal energy transmission. J. Sound Vib. 2019, 458. [CrossRef]

47. Macías-Díaz, J.E.; Motcheyo, A.B.T. Energy transmission in nonlinear chains of harmonic oscillators with long-range interactions. Results Phys. 2020, 18. [CrossRef]

48. Leon, J. Nonlinear supratransmission as a fundamental instability. Phys. Lett. A 2003, 319, 130-136. [CrossRef]

49. Leon, J.; Spire, A. Gap soliton formation by nonlinear supratransmission in Bragg media. Phys. Lett. A 2004, 327, 474-480. [CrossRef] 